\title{
Avaliação de ações municipais de combate a vetores da dengue na região de São José do Rio Preto, São Paulo, 1989 a 1995
}

\author{
Municipal activities against dengue vectors: a survey in the region of \\ São José do Rio Preto, São Paulo, 1989-1995 \\ Francisco Chiaravalloti Neto, Antônio I.P. da Costa, Maria Sílvia de Assis Moura, \\ Margareth R.D. Soares, Fernanda Correia Pereira, Marcos Battigaglia \\ e Francisco José $0 . S$. Aragão1
}

\begin{abstract}
Resumo $O$ objetivo do trabalho é medir as coberturas das atividades municipais de controle de Aedes aegypti e/ou Aedes albopictus, o casa-casa e o arrastão, realizadas entre 1989 e 1995 na região de São José do Rio Preto, São Paulo e avaliar a correlação cruzada entre elas e os Índices de Breteau (IB). Para o municípios com até 50.000 imóveis as coberturas conjuntas das atividade casa-casa e arrastão foram em sua maioria adequadas e as coberturas do casacasa apresentaram correlação cruzada negativa com os IB. Para município sede (maior que 50.000 imóveis) estas coberturas não apresentaram correlação com os IB. Em geral as coberturas foram inversamente proporcionais ao tamanho dos municípios. Para todas as faixas de tamanho de municípios, os arrastões não apresentaram correlação com os IB, mostrandose ineficazes.
\end{abstract}

Palavras-chaves: Aedes aegypti. Aedes albopictus. Municipalização. Controle. Erradicação.

\begin{abstract}
The aim of this study was to determine the coverage of municipal activities in terms of the control of Aedes aegypti and/or Aedes albopictus by routine house-to-house visits and by emergency activity, carried out between 1989 and 1995 in the area of São José do Rio Preto, São Paulo State, and to evaluate the cross-correlation between them and the Breteau index (BI). For towns with up to 50,000 real estate properties, the joint coverage by routine and emergency activities was mostly appropriate and the routine activities showed a negative cross-correlation with the $\mathrm{Bl}$. For the county seat (more than 50,000 real estate properties), the coverage provided by the above activities was not correlated with the BI. In general, the coverage was inversely proportional to town size. Emergency activities did not show a correlation with the BI in any town size range, proving to bes ineffective.
\end{abstract}

Key-words: Aedes aegypti. Aedes albopictus. Municipalization. Control. Eradication.

Tendo como base a perspectiva de que o controle do Aedes aegypti e/ou Aedes albopictus deveria estar baseado na integração das diversas instâncias responsáveis, a Superintendência de Controle de Endemias (SUCEN), propôs a partir de 1986 a assinatura de contratos de prestação de serviços com duração de 3 a 4 meses com Prefeituras de municípios do estado de São Paulo infestados pelos vetores da dengue e da febre amarela. O objetivo era que o município desenvolvesse atividades de controle dos vetores, principalmente a realização de visitas a

\footnotetext{
1 Superintendência de Controle de Endemias e Centro Universitário de Rio Preto, São José do Rio Preto e Laboratório de Estatística Aplicada do Departamento de Estatística da Universidade Federal de São Carlos, São Carlos, SP.

Endereço para correspondência: Dr. Francisco Chiaravalloti Neto. R. Rio Negro 160, Aclimação, 15091-390 São José do Rio Preto, SP. E-mail: fcneto@riopreto.com.br

Recebido em 24/6/98.
} 
todos os imóveis urbanos para retirada ou tratamento dos criadouros dos mosquitos e para orientação aos moradores. Esses contratos de curta duração abrangiam os meses de pico de infestação e foram custeados pelo Governo Estadual.

$\mathrm{Na}$ Região de São José do Rio Preto, no período de 1986 a 1988, foram firmados 22 contratos entre a SUCEN e Prefeituras Municipais, que apesar da curta duração, serviram de base para a introdução das atividades municipalizadas de controle de $A$. aegypti e/ou $A$. albopictus a partir de 1989 (SUCEN: dados não publicados).

No processo de implantação dos convênios do Sistema Unificado e Descentralizado de Saúde, no Estado, em 1989, foram previstos recursos para a realização de atividades municipalizadas de controle de vetores, em moldes semelhantes às executadas durante a vigência dos contratos, mas de forma ininterrupta e com periodicidade trimestral. Em 1989, 83\% dos municípios da região constituíram equipes municipais para estas atividades, chegando a 100\% em 19906.

Apesar do repasse de verbas inicial ter sido interrompido e só retomado episodicamente, os municípios acabaram mantendo, mesmo que de forma incipiente, a atividade municipalizada na região em função do risco e mesmo da ocorrência de epidemias de dengue. Isso ocorreu também em função da implantação do Sistema Único de Saúde (SUS) no estado de São Paulo, sendo o controle de vetores, uma das responsabilidades dos municípios.
A atividade municipal de controle de vetores a partir da sua implantação em 1989 foi desenvolvida basicamente de duas maneiras: as visitas sistemáticas e periódicas a todos os imóveis dos municípios por funcionários treinados, chamada de atividade casa-casa, e as atividades emergenciais realizadas em curto espaço de tempo por um contingente maior de pessoas, nos inícios dos verões e em momentos de altos índices de infestação ou com transmissão de dengue, chamados arrastões.

Em março de 1997 foi apresentada a versão final do Plano Diretor de Erradicação do Aedes aegypti no Estado de São Paulo, com o objetivo de ampliar a gama de atividades executadas pelos municípios, mas baseando-se no trabalho até então desenvolvido, isto é, a realização de visitas periódicas e ininterruptas aos imóveis para eliminação e/ou tratamento dos criadouros do mosquito7. Esta nova proposta, que representa uma mudança radical de estratégia, supõe que os municípios possam realizar adequadamente as atividades de combate aos vetores a ponto de ocorrer no futuro a eliminação do vetor.

Considerando as atividades municipais de combate ao vetor como um dos pilares de qualquer estratégia para evitar a ocorrência de epidemias de dengue, é objetivo deste trabalho medir as coberturas destas atividades, realizadas entre 1989 e 1995 e avaliar a existência de correlação cruzada entre elas e as mediadas de densidades larvárias realizadas no período.

\section{MATERIAL E MÉTODOS}

A região de São José do Rio Preto situa-se a noroeste do Estado de São Paulo e, até 1995 era constituída de 92 municípios. Em termos de colonização pelos vetores da dengue e febre amarela a primeira detecção de $A$. aegypti ocorreu em 19853, e a de A. albopictus em 19914. Atualmente, encontra-se infestada totalmente pelo $A$. aegypti e parcialmente pelo $A$. albopictus (SUCEN: dados não publicados).

Para efeito de análise, neste trabalho os municípios foram agrupados em quatro faixas de número de imóveis: de 1 a 1.000; de 1.001 a 5.000 ; de 5.001 a 50.000 ; e 50.001 e mais imóveis. Foram calculadas mensalmente para cada município as coberturas das atividades municipalizadas de controle, dividindo-se os imóveis visitados em cada mês pelo número total de imóveis da sua área urbana, multiplicandose o resultado por 100 .

Para cada faixa de tamanho de município, foram calculadas as coberturas médias mensais com a obtenção de séries históricas para o período de 1989 a 1995 para as atividades casacasa e arrastão, avaliando-se o comportamento das curvas para cada faixa de municípios e realizando-se a comparação das curvas entre todas as faixas.

A avaliação da infestação vetorial foi feita com base no Índice de Breteau (IB)2, obtido através de levantamentos amostrais realizados periodicamente em cada município1. O IB é calculado dividindo-se o número de recipientes positivos para larvas de $A$. aegypti e/ou $A$. albopictus pelo número de imóveis 
pesquisados, sendo o resultado multiplicado por 100. Para cada uma das faixas de municípios foram calculadas as médias mensais dos IB, obtendo-se também uma série histórica de valores médios mensais para o período de 1989 a 1995.

As séries de casa-casa e arrastões foram correlacionadas com as séries de IB utilizandose a técnica estatística de Modelos de Função de Transferência para Séries Temporais8.

Denominam-se Modelos de Função de Transferência aqueles que relacionam uma série de tempo de saída com uma série de tempo de entrada8, assumindo que $x_{t}$ e $y_{t}$ sejam séries estacionárias. O modelo, ou função, que relaciona estas duas séries é o objetivo da análise estatística. Esta análise consiste em identificar, estimar e verificar um modelo. Existem suposições matemáticas para que o modelo, após identificado, possa ser estimado. Como todo modelo, acrescenta-se um termo de erro aleatório que deve ser independente da série de entrada. Os coeficientes do modelo são chamados pesos de impulso de resposta. A série de saída só é afetada pela série de entrada através de valores passados e atual.

O modelo matemático apresentado acima pode ser escrito como:

$$
y_{t}=v_{0} x_{t}+v_{1} x_{t-1}+v_{2} x_{t-2}+\ldots+\eta_{t}=v(B) x_{t}+\eta_{t}
$$

onde : $\eta_{\mathrm{t}}$ é ruído branco, erro aleatório com média zero, variância constante e não correlacionado com os seus valores passados;

$y_{t}$ é a série de saída;

B: operador passo atrás

$x_{t}$ é a série de entrada;

$v(B)=\sum_{j=-\infty}^{\infty} v_{j} B_{j}, \Sigma\left|v_{j}\right|<\infty$;

$x_{t}$ e $\eta_{t}$ são independentes.

O objetivo do modelo de função de transferência é estimar $v(B)$. Seguindo a metodologia de Box-Jenkins dividimos o ajuste desse modelo em 3 etapas: $1^{\underline{a}}$ ) identificação do modelo; $2^{\underline{a}}$ ) estimação do modelo; $3^{a}$ ) verificação do modelo.

A 1 a etapa é feita baseada na função de correlação cruzada amostral. Na $2^{-a}$ etapa, após identificar um possível modelo, a estimação é feita através do método da máxima verossimilhança. $\mathrm{Na} 3^{\mathrm{a}}$ etapa a verificação do modelo é feita analisando-se a série dos resíduos. Se o modelo for totalmente adequado a série de resíduos será ruído branco, e não será correlacionada com a série de entrada, isto pode ser verificado através da função de correlação cruzada dos resíduos com a série de entrada. Caso o resíduo não seja ruído branco, o modelo pode também ser considerado adequado, mas sugere-se a inclusão de outras variáveis neste modelo. Para ajuste dos modelos de função de transferência foi utilizado o pacote estatístico SAS.

\section{RESULTADOS}

Na Figura 1, estão apresentadas as coberturas médias mensais das atividades casa-casa por faixa de número de imóveis. Para os municípios entre 1 e 1.000 imóveis, a quase totalidade das coberturas ficou entre 40 e $90 \%$, sendo que em geral variou na faixa de 40 a $60 \%$, mostrando que a meta estabelecida de visitar mensalmente pelo menos um terço dos imóveis foi cumprida e em muitos casos ultrapassada. Para os municípios na faixa de 1.001 a 5.000 imóveis a grande maioria das coberturas médias mensais ficou entre 20 e $40 \%$, mostrando também uma adequação da atividade à meta estabelecida.

Para os municípios na faixas de 5.001 a 50.000 imóveis as coberturas variaram em geral entre 10 e $30 \%$, não se verificando a adequação encontrada nos casos anteriores. Na faixa com mais de 50000 imóveis inclui-se apenas o município sede da região, São José do Rio Preto, apresentando, em geral coberturas inferiores a $20 \%$, com grande número de valores inferiores a $10 \%$ ou mesmo iguais a zero, mostrando baixa adequação à meta estabelecida.

As coberturas médias mensais da atividade arrastão para as várias faixas de municípios são apresentadas nas Figuras 2 e 3 . Nota-se que estas atividades passaram a ser desenvolvidas a partir do final de 1989 e, em geral, com maior intensidade entre os meses de outubro a maio, e que as coberturas apresentaram maior oscilação do que nas atividades casa-casa. As coberturas médias variaram entre 0 e $60 \%$ para os municípios até 1.000 imóveis, atingindo-se até valores iguais a $100 \%$, entre 0 e $40 \%$ para os municípios entre 1.001 e 5.000 imóveis, e entre 0 e $30 \%$ para aqueles entre 5.001 e 50.000 imóveis. Para o município de São José do Rio Preto os valores foram em geral iguais a zero, 


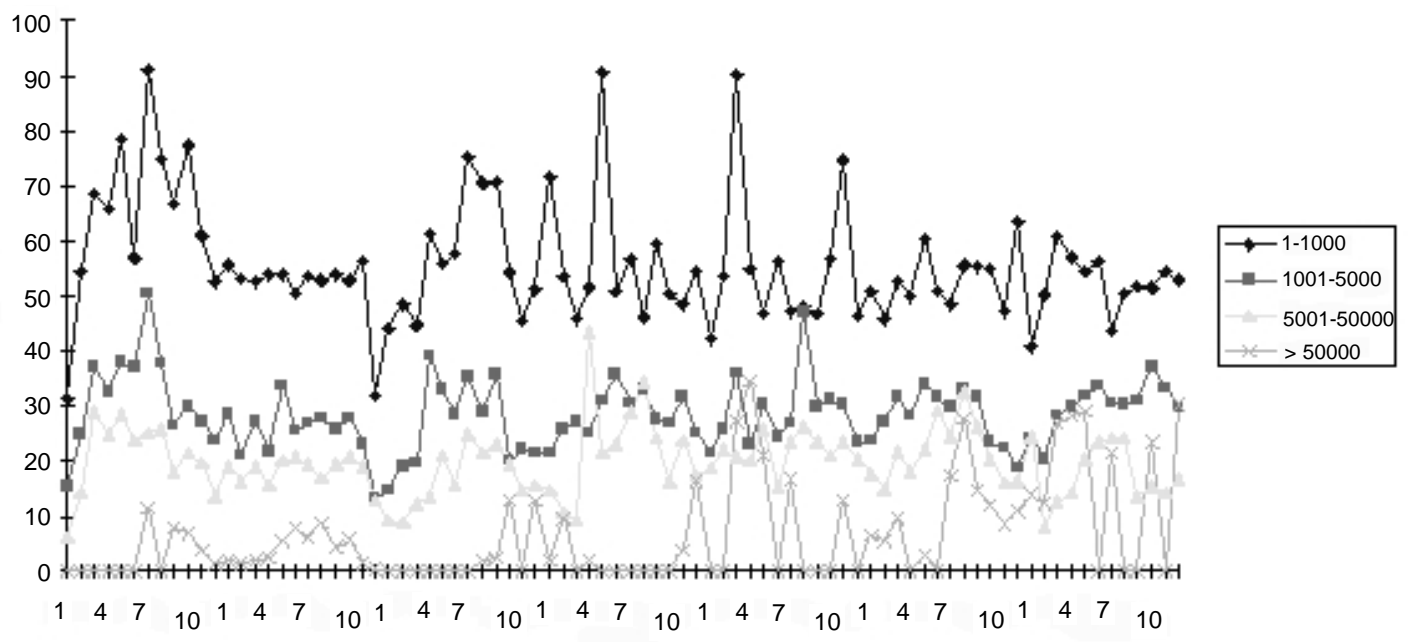

Figura 1 - Coberturas médias mensais em porcentagens das atividades casa-casa, segundo meses e faixas de tamanho de municípios por número de imóveis, região de São José do Rio Preto, janeiro de 1989 a dezembro de 1995.

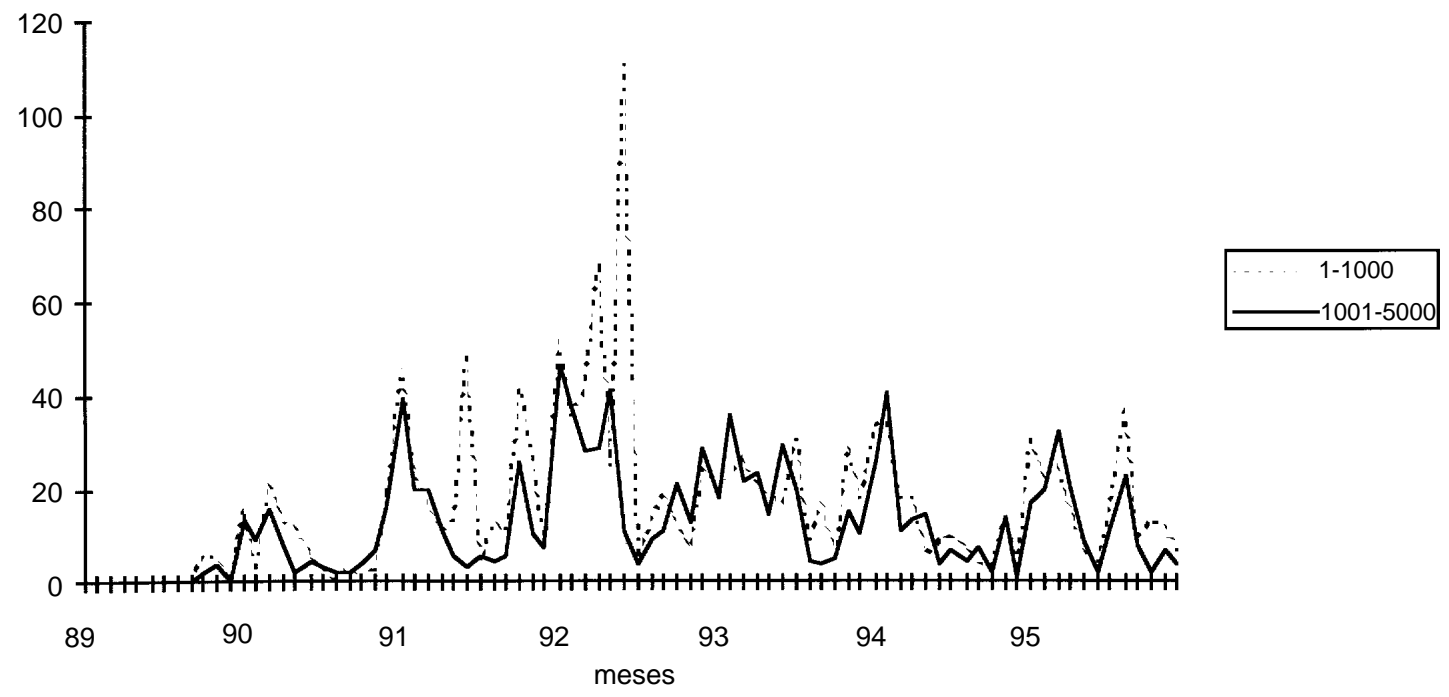

Figura 2 - Coberturas médias mensais dos arrastões, segundo meses e municípios de 1 a 1000 imóveis e 1001 a 5000 imóveis, região de São José do Rio Preto, janeiro de 1989 a dezembro de 1995.

com a apresentação de alguns picos nos verões.

Após os ajustes dos modelos de função de transferências, verificou-se, para os municípios com até 1.000 imóveis e entre 5.001 a 50.000, a existência de correlação cruzada negativa entre a cobertura do casa-casa e o IB, porém o resíduo não foi ruído branco, indicando a existência de outras variáveis que deveriam ser consideradas no modelo para explicar o IB. Para os municípios entre 1.001 e 5.000 imóveis, verificou-se também a existência de correlação cruzada negativa entre 


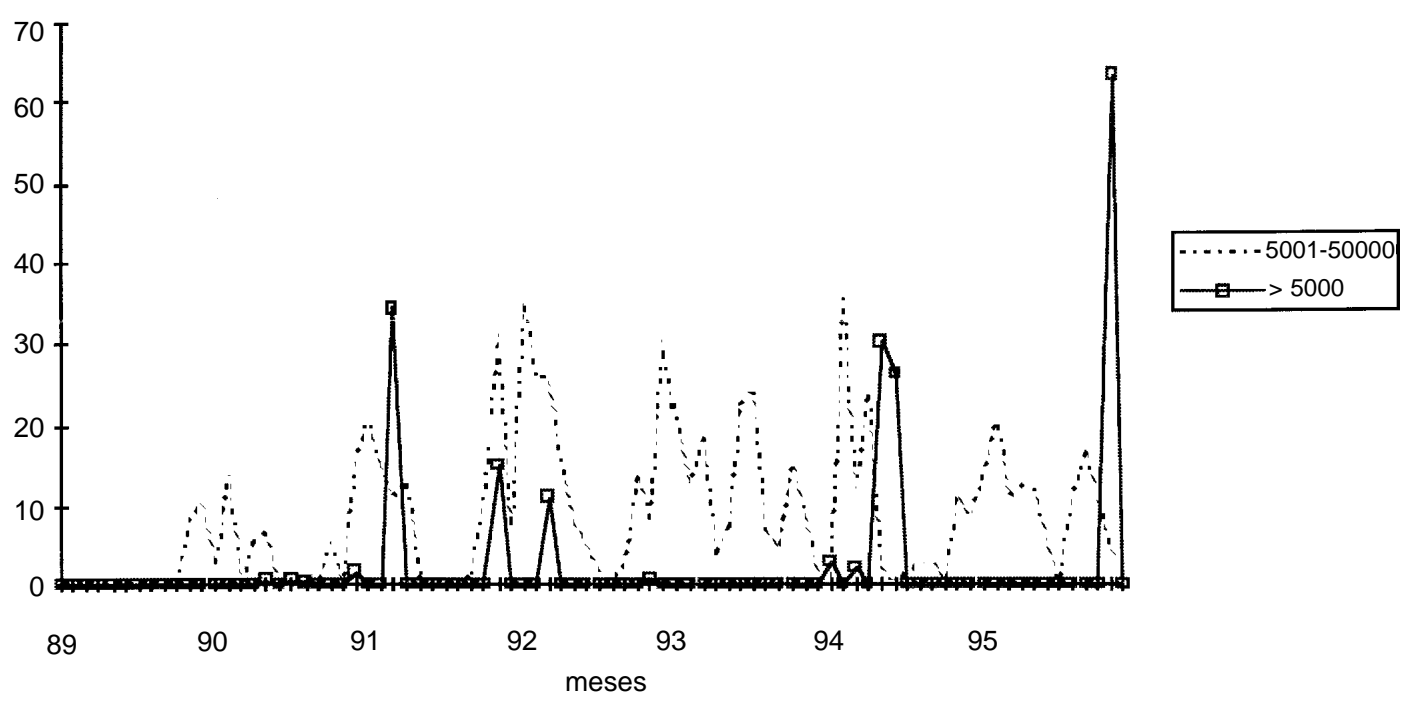

Figura 3 - Coberturas médias mensais dos arrastões, segundo meses e municípios de 5001 a 50000 imóveis e com mais de 50000 imóveis, região de São José do Rio Preto, janeiro de 1989 a dezembro de 1995.

as duas variáveis, sendo o modelo bem ajustado, isto é, o que melhor prevê o IB. Para o município sede verificou-se que a cobertura casa-casa não apresentou correlação cruzada com o IB, sendo estas variáveis consideradas independentes.
Para todas as faixas de tamanho de municípios não se verificou a existência de correlação cruzada entre a cobertura arrastão e o IB, isto é, a cobertura não afetou o IB, podendose dizer que as variáveis são independentes.

\section{DISCUSSÃO}

Considerando-se as atividades casa-casa e arrastão, pode-se afirmar que, com exceção do município sede, a meta de realização de uma visita trimestral a todos os imóveis foi alcançada totalmente ou em grande parte pela maioria dos municípios. Por outro lado, constatou-se uma relação inversamente proporcional entre tamanho de município e coberturas, apontando para dificuldades operacionais crescentes quanto maior for o número de imóveis.

Das atividades realizadas, somente o casacasa mostrou-se correlacionado negativamente com os níveis de infestação para municípios até 50.000 imóveis. O mesmo não ocorreu com os arrastões, atividades que passaram a ser incentivadas a partir do final de 1989 , principalmente nos meses quentes e chuvosos e em situações com altos níveis de infestação dos vetores ou com epidemias. Supunha-se que estas atividades emergenciais, realizadas rapidamente, poderiam causar uma diminuição importante do número de criadouros dos vetores. O fato de não terem afetado os IB, vem mostrar que não produziram resultados palpáveis, devendo ser melhor analisadas e repensadas. Por exemplo, um estudo que quantificasse para a região os tipos de recipientes mais importantes para os vetores da dengue, poderia esclarecer a falta de relação verificada entre estas atividades e os IB. Uma possível suspensão dos arrastões acompanhada de um aprimoramento do casacasa é uma possibilidade que merece ser discutida.

Mesmo com as coberturas alcançadas pelas atividades municipais de controle dos vetores da dengue e da existência de correlação cruzada negativa entre as atividades casa-casa e os IB para os municípios com até 50.000 imóveis, epidemias de dengue vem ocorrendo nos municípios maiores e menores da região, mostrando que, se houve algum efeito, essas atividades não foram suficientes para eliminar o risco de epidemias. Por outro lado a manutenção de um contingente de funcionários nos municípios 
é fundamental para o sucesso de qualquer estratégia a ser adotada. Se o trabalho não viesse sendo executado desde 1989, talvez a transmissão de dengue ocorresse em maiores proporções do que os níveis atuais (Secretaria Estadual de Saúde de São Paulo: dados não divulgados).

Apesar dos resultados encontrados para o casa-casa, supervisões realizadas em campo têm mostrado que esta atividade está sujeita a uma série de problemas, como: a) a falta constante de funcionários, principalmente nos municípios maiores; b) os desvios de funções dos funcionários para outras atividades; c) a falta de viaturas, treinamentos e integração com outras atividades como o controle de insetos incômodos, a vigilância sanitária e o controle de roedores; e d) a rotina repetitiva a que são submetidos os funcionários, fazendo com que nem eles próprios e nem os moradores valorizem este tipo de atividade (SUCEN: informações não publicadas).

Outra questão importante a ser levantada é que as visitas domiciliares deveriam ser melhor aproveitadas para um relacionamento mais aprofundado entre os funcionários municipais e os moradores. Estes deveriam, necessariamente, acompanhar o trabalho, identificando os problemas e as soluções. A prioridade deveria ser dada ao incentivo à participação do morador no combate ao vetor. A atividade casa-casa não pode ser vista isoladamente, e nem baseada somente na aplicação de larvicidas, e só poderá funcionar na medida que incentive a participação comunitária.

Dentro de uma perspectiva de controle ou de erradicação, a atividade casa-casa é de grande importância e deve ser incentivada, mas há a necessidade da realização de um grande esforço e investimento para a melhoria de sua qualidade e eficácia, para que novas epidemias de dengue possam ser evitadas.

As atividades de controle da dengue no Estado de São Paulo, seguindo uma mesma tendência identificada por Gratz 5 em outros países, prescindem da realização de estudos de avaliação que indiquem as direções a serem seguidas. Nesse sentido, a mudança da estratégia de controle para a erradicação deveria ser precedida por uma série de atividades de pesquisa que corroborassem o caminho a ser seguido. Este trabalho faz uma tentativa de avaliar as atividades municipais desenvolvidas até aqui, procurando contribuir para a discussão e o equacionamento do problema da transmissão da dengue.

\section{AGRADECIMENTOS}

Aos funcionários Beatriz A.C. Belini, Dora B. Defende, Marcelo D. Papa, Neusa de Fátima A. Santana, Perpétua M. Magalhães e Ruth Neide
A.B. da Silva, pela colaboração no levantamentos dos dados.

\section{REFERÊNCIA BIBLIOGRÁFICAS}

1. Alves MCP, Gurgel SM, Almeida MCRR. Plano amostral para cálculo de densidade larvária de Aedes aegypti e Aedes albopictus no Estado de São Paulo, Brasil. Revista de Saúde Pública 25: 251-256, 1991.

2. Breteau H. La fievre jaune en Afrique-occidentale Francaise. World Health Organization Bulletin 11:453-481, 1954.

3. Chiaravalloti Neto F. Descrição da colonização de Aedes aegypti na região de São José do Rio Preto, São Paulo. Revista da Sociedade Brasileira de Medicina Tropical 30:279-285, 1997.

4. Chiaravalloti Neto F, Costa AI, Soares MR, Scandar SA, Cardoso Junior RP. Descrição da colonização de Aedes albopictus (Diptera: Culicidae) na região de São José do Rio Preto, SP, 1991-1994. Revista da Sociedade Brasileira de Medicina Tropical 26:543-548, 1996.
5. Gratz NG. Lessons of Aedes aegypti control inThailand. Medical and Veterinary Entomology 7:1-10, 1993.

6. Lo SS. Epidemiologia e controle do dengue no Estado de São Paulo. Avaliação do processo de municipalização das atividades de controle do Aedes aegypti na Região de Presidente Prudente, 1985 a 1991. Tese de Mestrado Faculdade de Ciências Médicas da Universidade Estadual de Campinas, Campinas, 1993.

7. Superintendência de Controle de Endemias. Secretaria de Estado da Saúde. Plano Diretor de Erradicação do Aedes aegypti no Estado de São Paulo. São Paulo [mimeo], 1997.

8. Wei, WS. Time Series Analysis. Univariate and multivariate methods. Addeson Wesley Publishing Co., Redwood City, 1990. 